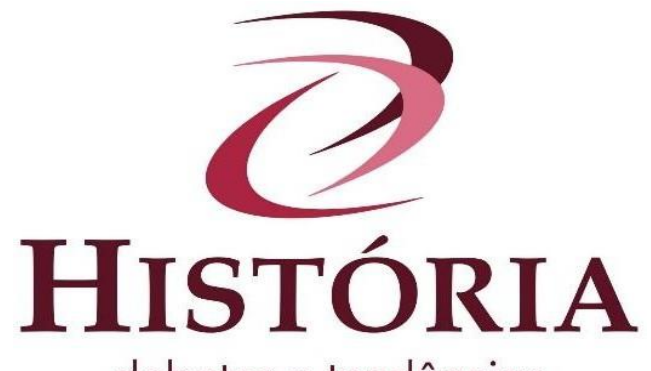

debates e tendências

\title{
Do projeto unificador à fragmentação prática: a cancerologia e os cancerologistas no Brasil (1930 - 1970).
}

\section{From the unifying project to practical fragmentation: cancerology and cancerologists in Brazil (1930 - 1970).}

\section{Del proyecto unificador a la fragmentación práctica: la cancerología y los cancerólogos en Brasil (1930 - 1970).}

Luiz Alves Araújo Neto ${ }^{i}$ Luiz Antonio Teixeirai

Resumo: Este artigo discute o processo de especialização médica em torno do câncer no Brasil entre os anos 1930 e 1970, especificamente o projeto de desenvolvimento de uma especialidade que reuniria conhecimentos e práticas no combate à doença, a cancerologia. Inicialmente parte do movimento pela campanha nacional contra o câncer, gradualmente a cancerologia ganhou contorno de especialidade, com propostas para titulação e inclusão de disciplinas nos currículos médicos. Pensada como uma especialidade unificadora, a cancerologia teve como desfecho uma prática fragmentada no interior de diversas especialidades. Argumentamos que esse processo de fragmentação ocorreu devido à especialização de outras especialidades médicas, principalmente quanto à incorporação tecnológica, inviabilizando o projeto unificador da cancerologia.

Palavras-chave: cancerologia; especialidade; especialização; história da medicina; câncer.

\begin{abstract}
This article discusses the process of medical specialization on cancer in Brazil between the 1930s and the 1970s, specifically the project to develop a specialty that would bring together knowledge and practices to fight against the disease, named cancerology. Initially part of the movement for the national campaign against cancer, cancerology gradually became a specialty, with proposals for the titling and inclusion of disciplines in medical curricula. Conceived as a unifying specialty, cancerology ended up with a fragmented practice within several specialties. We argue that this fragmentation process occurred due to the specialization of other medical specialties, mainly regarding technological incorporation, making the unifying project of cancerology unfeasible.
\end{abstract}

Keywords: cancerology; specialty; specialization; history of medicine; cancer.

Resumen: Este artículo trata del proceso de especialización médica en torno al cáncer en Brasil entre los años 1930 y 1970, específicamente el proyecto de desarrollo de una especialidad que reuniría conocimientos y prácticas en la lucha contra la enfermedad, la cancerología. Inicialmente parte del movimiento de la campaña nacional contra el cáncer, poco a poco la cancerología adquirió un enfoque de especialidad, con propuestas de titulación e inclusión de disciplinas en los planes de estudio de medicina. Pensada como una especialidad unificadora, la cancerología tuvo como resultado una práctica fragmentada. Sostenemos que este proceso de fragmentación se produjo debido a la especialización de otras especialidades médicas, principalmente en cuanto a la incorporación tecnológica, haciendo inviable el proyecto unificador de la cancerología. 
Palabras clave: cancerología; especialidad; especialización; historia de la medicina; cáncer.

\section{Introdução}

Em 1935, a Sociedade de Medicina e Cirurgia do Rio de Janeiro, em comemoração do seu cinquentenário, realizou o Primeiro Congresso Brasileiro de Câncer, reunindo médicos e atores do campo político engajados na organização da "luta anticancerosa" no país. Entre os participantes do congresso, constavam personagens que tiveram atuação central na criação das primeiras instituições de cuidado dedicadas especificamente aos tumores malignos. Além de advogar pela criação de um hospital para cancerosos no Rio de Janeiro e pela criação de uma campanha nacional contra o câncer (Teixeira, 2009), os presentes também compartilhavam a construção gradual de um papel social, a figura de cancerologista, o principal especialista na doença.

De modo geral, os conhecimentos e práticas médicas eram bastante limitados para lidar com o câncer no início do século XX, num ambiente permeado por incertezas quanto à etiologia da doença, ao seu mecanismo patológico, às possibilidades diagnósticas e à capacidade terapêutica (Araújo Neto, 2019). A emergência desses indivíduos autointitulados cancerologistas correspondia, em parte, à necessidade de estabelecimento de credibilidade da medicina e de articulação política para a criação de instituições e financiamento do Estado (Teixeira e Fonseca, 2007). A construção da figura do cancerologista também se vinculou aos processos de especialização médica e demarcação de especialidades como coletivos de práticas e de poder.

Abordado como uma doença local desde a segunda metade do século XIX ${ }^{\mathrm{iii}}$, o câncer foi objeto de diferentes especialidades, como a ginecologia, a dermatologia e a cirurgia. $\mathrm{O}$ cuidado fragmentado entre vários especialistas, para os cancerologistas, atrapalhava a compreensão clínica do adoecimento e a organização da luta contra o câncer ${ }^{\text {iv }}$. Dessa forma, a cancerologia surgia como uma especialidade que unificaria os conhecimentos e práticas para lidar com os tumores, formando um "especialista completo". Porém, a proposta de unificação enfrentava resistências em virtude de o processo mais geral de especialização da medicina estar relacionado à localização corporal dos agravos e aos sistemas corpo humano. Nesse contexto, o câncer como um conjunto de doenças diferenciadas pela localização no corpo e morfologia tendia a ser fragmentado entre diversas especialidades.

Este artigo discute a história da especialização médica em torno do câncer no Brasil, especificamente a emergência e arrefecimento do projeto de institucionalização da cancerologia como especialidade, processo que pode ser compreendido entre 1930 e 1970. 
Exploramos aqui dois argumentos. O primeiro é que a especialização médica em torno do câncer no Brasil teve como vetor principal o diagnóstico precoce, o que diferencia o processo em relação a países europeus e da América do Norte, onde a inovação terapêutica foi o motor principal (Pickstone, 2008; Hayter, 2004). O segundo argumento deste artigo é que a fragmentação da cancerologia teve como fator principal a incorporação de novas tecnologias diagnósticas e seu impacto na composição das especialidades que já lidavam com o câncer na prática cotidiana.

A discussão desenvolvida neste artigo dialoga com diferentes vertentes da história e sociologia da medicina, principalmente com os trabalhos do historiador George Weisz (2003; 2006) e dos sociólogos Patrice Pinell (2010) e Bernike Pasveer (1989). A análise também é parte de um esforço mais amplo de pesquisa sobre a história do câncer no Brasil, pela qual já foram exploradas a trajetória das políticas de controle da doença (Teixeira e Fonseca, 2007), a emergência dos tumores na agenda da medicina brasileira (Teixeira, 2009), a relação entre câncer, civilização e desenvolvimento (Araújo Neto e Teixeira, 2017), a incorporação de tecnologias diagnósticas ao sistema de saúde (Teixeira e Araújo Neto, 2020a), entre outros temas.

Para o presente estudo, abordamos diferentes fontes, sobretudo publicações médicas, como a Revista Brasileira de Cancerologia (Rio de Janeiro), os Arquivos de Oncologia (Salvador) e o Boletim de Oncologia (São Paulo); anais de congressos; livros especializados; documentos de arquivo; além de documentação política, como decretos e leis.

\section{Especialização e especialidades médicas como objetos historiográficos}

Um dos fenômenos fundamentais relacionados ao desenvolvimento profissional e cognitivo da medicina nos séculos XIX e XX foi o processo de especialização. Uma historiografia mais tradicional da medicina adotou uma interpretação para a especialização como parte de uma grande linha evolutiva do pensamento médico, levando à necessidade de segmentá-lo para lidar com as novas doenças e os saberes relacionados a elas (Dachez, 2004). Abordagens mais recentes, porém, têm proposto uma discussão que articule a especialização à organização do trabalho médico, à atuação política da medicina, aos embates sobre autoridade das práticas e à inovação e incorporação tecnológica.

Na leitura de George Weisz, a especialização não está relacionada somente ao progresso do conhecimento, à divisão do trabalho, ou mesmo às demandas sociais criadas pela urbanização e industrialização, e sim, a um conjunto de fatores que não podem ser 
generalizados (Weisz, 2006). Antes de ter um modelo para o processo, o autor propõe a existência de condições essenciais para a especialização, quais sejam: 1) a unificação entre medicina e cirurgia; 2) o desenvolvimento do conhecimento médico; e 3) a emergência dos Estados Nacionais e sua primazia pela quantificação e gerenciamento da população (Weisz, 2003). Além dessas condições de possibilidade, também foi importante o enquadramento das doenças como entidades específicas, com vidas patológicas próprias e peculiaridades que mobilizassem campos para seu estudo, diagnóstico e tratamento (Rosenberg, 2002).

Patrice Pinell, por sua vez, argumenta que o surgimento de novas especialidades é um movimento ao mesmo tempo endógeno e exógeno ao campo médico, e altera as relações estabelecidas dentro e fora dele. Para ele, a definição de um projeto de "cancerologia", a princípio, esbarraria em dois fatores primordiais: a existência de um número considerável de áreas específicas que lidavam com a doença em clínicas e hospitais, reivindicando autoridade sobre tipos particulares de tumores, e o domínio de algumas especialidades na definição das políticas e ações coletivas de combate à doença, caso da radiologia na França (Pinell, 2010).

Esse movimento de afirmação da autoridade através do conhecimento científico possuía duas vias: por um lado, os cancerologistas buscavam se colocar em distinção aos “charlatães”, apresentados como ameaças aos pacientes, por não atenderem aos cânones da medicina científica $^{\mathrm{v}}$ (Figura 01). Por outro lado, ocorria também a demarcação da cancerologia como um conjunto específico de práticas, que, em meados do século, significava no Brasil a combinação da clínica cirúrgica à radiologia ${ }^{\text {vi }}$. 
Figura 01 - Cartaz de campanha educativa produzida pelo Serviço Nacional de Câncer (SNC), na década de 1940.

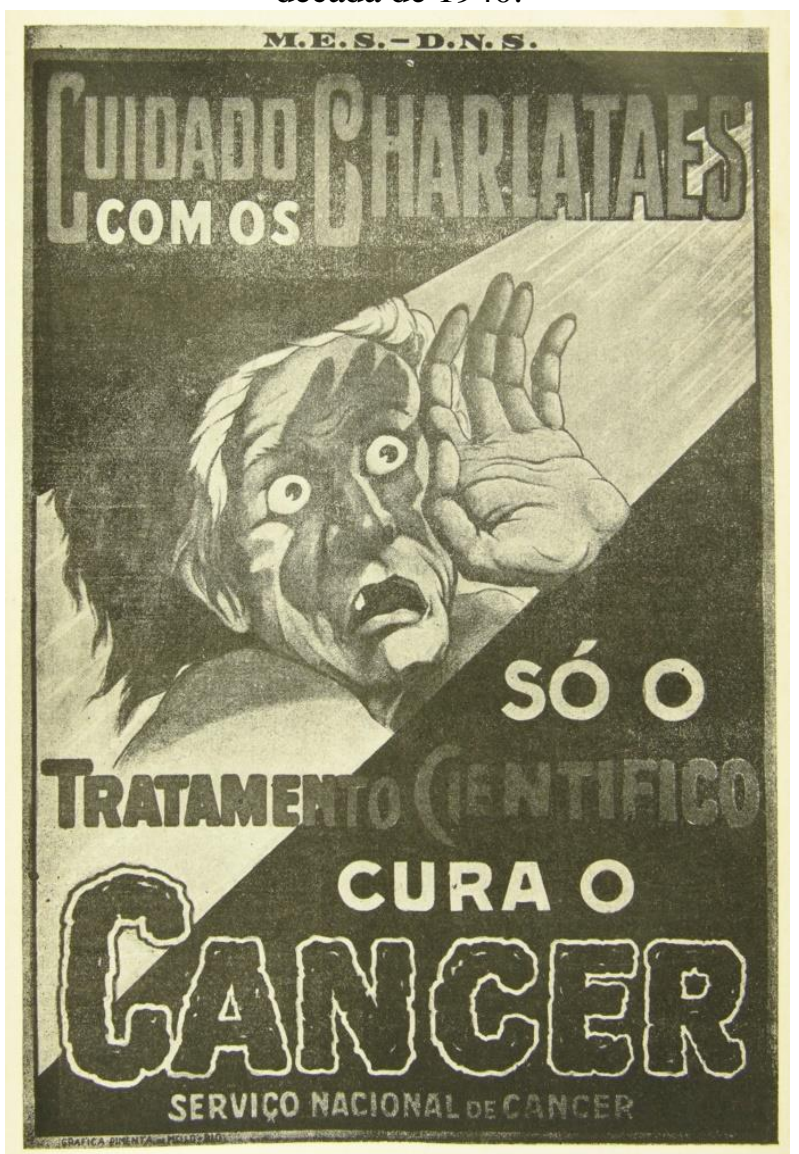

Acervo: Projeto História do Câncer/Fiocruz.

Da mesma forma, as discussões em torno de ferramentas diagnósticas e da etiologia da doença também demarcavam dimensões específicas da prática médica aos cancerologistas e às especialidades que atuavam conjuntamente a eles. É importante, nesse sentido, a argumentação de Pasveer (1989) acerca da relação entre especialização e inovação tecnológica. Na sua leitura, novas tecnologias não são simplesmente incorporadas pelos especialistas, mas também participam do processo de especialização, na medida em que moldam novas semiologias e práticas, por vezes mesmo reorganizando os próprios coletivos especializados.

Adotando as perspectivas desses autores, abordamos a tentativa de organização da cancerologia como uma especialidade situada nos contextos de transformação do câncer em problema de saúde pública, de articulação dos cancerologistas com atores de diferentes campos sociais, e de incorporação de novas tecnologias diagnósticas. Esse movimento teve início a partir de redes traçadas por atores bem posicionados em seus campos, cirurgiões que assumiram para si a alcunha de cancerologistas. 


\section{A campanha nacional contra o câncer e a primeira geração de cancerologistas}

Nas décadas de 1920 e 1930, o câncer despertava poucas mobilizações sociais, sendo considerada uma doença com baixa incidência no Brasil, principalmente por ser um problema de nações civilizadas, não do imenso hospital como era descrito o país (Araújo Neto \& Teixeira, 2017). Também nesse período não existiam grupos profissionais bem organizados para lidar com a doença, havendo inclusive debates acerca de quais seriam os espaços da prática médica capazes de oferecer respostas eficazes contra os tumores, a clínica, o laboratório ou a atuação pública da higiene (Araújo Neto, 2019). Apesar disso, alguns nomes importantes do campo médico brasileiro, como Eduardo Rabello, indicavam a necessidade de se organizar uma luta contra o câncer no país ${ }^{\text {vii }}$.

Durante os anos 1930, alguns médicos começaram a se organizar para propor ações de combate à doença, tendo como lideranças cirurgiões, a exemplo de Mário Kroeff e Antônio Prudente. Isso se devia, principalmente, à incorporação das cirurgias radicais às rotinas de cuidado dos cancerosos no país (Teixeira, 2009). Esses procedimentos tinham como característica principal incisões bastante amplas, retirando o tumor e todas as áreas circunvizinhas, com o intuito de evitar a metástase ${ }^{\text {viii }}$ (Kroeff, 1936; Prudente, 1939). Embora a eficácia dessas cirurgias, nos melhores cenários, possibilitasse até cincos anos de uma sobrevida dolorosa aos pacientes ${ }^{\mathrm{ix}}$, a chance de uma intervenção terapêutica não somente fortaleceu a ideia de uma luta coordenada contra o câncer, mas também ampliou a credibilidade dos cirurgiões.

Com o Primeiro Congresso Brasileiro de Câncer, realizado em 1935, e a implantação do Estado Novo de Vargas, em 1937, o movimento político para elaboração de uma campanha nacional ganhou força, resultando na criação do Centro de Cancerologia, em 1937, e do Serviço Nacional de Câncer (SNC), em 1941, ambos no Rio de Janeiro (Teixeira \& Fonseca, 2007). Nos anos 1940, as mobilizações pela luta anticancerosa se proliferaram pelos estados da federação, com pautas e lideranças específicas, mas buscando vínculo ao SNC. Nesse processo, outros cirurgiões assumiram para si esse papel de destaque, caso de Antônio Saint-Pastous (RS), Haroldo Juaçaba (CE), Napoleão Laureano (PB).

A construção dessas figuras de liderança passava pela definição do papel de um especialista em câncer, um personagem que pudesse não somente oferecer conhecimentos e práticas para o cuidado dos doentes, mas também pudesse ser porta-voz das mobilizações contra os tumores (Araújo Neto, 2018). É desse processo de elaboração de uma autoridade que resulta a figura do cancerologista no Brasil, um cirurgião que coordenava algum serviço 
ou seção de câncer e, em alguns casos, possuía especialização em cirurgia de tumores no exterior. Seria possível traçar uma primeira geração de cancerologistas a partir de três características: 1) a especialização em cirurgia; 2) o papel de destaque na organização de ações de combate; e 3) autointitulação do estatuto de cancerologista.

Um ponto importante nessa primeira geração diz respeito ao título. Mesmo intitulando a si mesmos como cancerologistas, esses especialistas não possuíam traços de distinção em relação a outros cirurgiões, ou mesmo espaços institucionais dedicados à cancerologia. Na realidade, parte do movimento de construção das instituições de cuidado envolveram a criação de cargos específicos nos novos hospitais.

A construção da figura do cancerologista no Brasil teve como referência as atuações de Mário Kroeff e Antônio Prudente. Ambos cirurgiões e lideranças no movimento por uma campanha nacional contra o câncer, não somente assumiram o papel de porta-vozes das ligas e associações, mas também propuseram modelos para organização do combate à doença. Outro aspecto relevante em suas atuações foi a defesa da cirurgia, mais especificamente a eletrocirurgia, como método terapêutico principal no tratamento dos cancerosos (Teixeira, 2010; Araújo Neto, 2018b).

A partir de suas atuações, a figura do cancerologista gradativamente foi contornada por um projeto de especialização. Para que a luta contra o câncer pudesse avançar no país, era necessário formar outros especialistas, ampliar o contingente de médicos capazes de lidar com a doença ${ }^{\mathrm{x}}$. Nesse sentido, demandava-se a produção de conhecimento sobre os tumores, a incorporação de uma disciplina específica para o câncer nos currículos das escolas médicas, e a criação de espaços institucionais dedicados ao cuidado dos doentes. A viabilização da campanha nacional contra o câncer, na visão dos primeiros cancerologistas, estava intrínseca à existência de uma especialidade dedicada exclusivamente à doença. Nos anos 1940, com a criação do Serviço Nacional de Câncer, o projeto da cancerologia ganhou novos contornos.

\section{O projeto unificador da cancerologia nos anos 1940 e 1950}

Em 1946, foi criada a Sociedade Brasileira de Cancerologia, com o intuito de levar adiante a discussão sobre a especialização médica. Fundada pelos membros do Serviço Nacional de Câncer, a Sociedade teve o início de sua trajetória bastante imbricada ao SNC. Em 1947, como previsto no regimento de criação do Serviço, foi lançada a Revista Brasileira de Cancerologia, primeira publicação dedicada exclusivamente ao câncer no Brasil, com o objetivo inicial de divulgar as ações do Centro de Cancerologia e receber 
trabalhos de médicos de outros estados do país. O comitê editorial da revista, presidido por Mário Kroeff, acreditava ser possível articular uma rede de atores especializados na doença através do periódico ${ }^{\mathrm{xi}}$.

A grande expectativa dos primeiros cancerologistas estava na obtenção de recursos via governo federal para a efetivação da campanha nacional contra o câncer. O número de associações e ligas se multiplicaram ao longo dos anos 1940, e os médicos em cada estado se articulavam para obter recursos com as elites locais e comprovar a importância do câncer na agenda pública. Em paralelo, novas pesquisas no campo da biomedicina começavam a transformar a predominância do estilo de pensamento anatomoclínico quanto à etiologia do câncer. O questionamento da origem da doença pelo traumatismo ganhou força com os trabalhos que discutiam a carcinogênese (processo de formação dos tumores) e a contribuição de certas substâncias nesse processo.

A questão da especialização em cancerologia ganhava considerável importância nesse cenário. Em 1952, Kroeff colocou a discussão em pauta na Revista Brasileira de Cancerologia, defendendo a necessidade de incluir o tema no currículo médico das faculdades. A base de sua argumentação consistia na especificidade da prática "cancerológica", tanto no diagnóstico quanto no tratamento, pois lidava com grande diversidade de tumores e com um problema de difícil abordagem. Segundo Kroeff:

\footnotetext{
No diagnóstico, não só a clínica cancerológica requer adestramento profissional no reconhecimento da doença que pode se localizar em qualquer parte do organismo, como exige o hábito do microscópico para confirmar e diferenciar a patologia das lesões, estas mesmas com variada indicação nos processos terapêuticos. Não só a experiência no exame dos doentes e manejo do microscópio se tornam necessários, mas também a endoscopia aplicada, num e noutro órgão, é indispensável à prática da cancerologia moderna, sem falar na tomada das biópsias, que só fazem bem àqueles que estão familiarizados com o exercício da clínica especializada na doença ${ }^{x i i}$.
}

Para ele, a complexidade da abordagem à doença, aliada à "importância atribuída hoje ao mal", tornava evidente a inclusão da cancerologia no currículo médico. Com base no caminho traçado nos dez anos anteriores, da fundação do SNC ao momento de sua argumentação, Kroeff defendia que o estatuto social do câncer justificava um maior investimento social na cancerologia. Se, desde o início da campanha, a ideia de que somente um profissional capacitado poderia identificar corretamente e precocemente a doença fora veiculada fortemente em cartazes, jornais, palestras, e mesmo publicações médicas, era necessário delimitar claramente como seria a formação desse médico.

Com base nos argumentos colocados por Kroeff, e a partir da constatação de que grande quantidade de profissionais não sabia como lidar com a doença na prática clínica, o 
Serviço Nacional de Câncer utilizou de sua prerrogativa de organizador da campanha nacional para realizar cursos de formação em cancerologia, iniciados em 1953. Cursos sobre a doença já existiam no país há alguns anos, sendo oferecidos pontualmente por cirurgiões considerados referência na prática clínica, como o professor da Faculdade de Medicina da Universidade do Brasil, Fernando Paulino, ou mesmo o médico do SNC, Döllinger da Graça e o cirurgião Ugo Pinheiro Guimarães. Apesar disso, não havia a preocupação clara com a formação do cancerologista, o médico que deveria diagnosticar e tratar o câncer.

Os cursos fornecidos pelo SNC tinham como objetivo atualizar profissionais já experientes na área e apresentar elementos básicos sobre os tumores. Em algumas edições, os cursos possuíam grades bastante específicas, como o ministrado em 1955, que abordava principalmente os tumores ginecológicos. Esses momentos de formação também eram dedicados a divulgar as expectativas e projetos da campanha nacional, dada a presença de médicos de diversos estados na composição das turmas ${ }^{x i i i}$.

O desenvolvimento da cancerologia e a mobilização pela campanha contra o câncer estiveram imbricados, pois os atores envolvidos no primeiro buscavam legitimá-lo através de ações do segundo, e vice-versa. Um exemplo concreto disso foi a realização, em São Paulo, em 1954, do VI Congresso Internacional de Câncer, organizado pela Union Internationale Contre le Cancer (UICC) e tendo como patrono o então diretor do SNC, Antônio Prudente. O evento reuniu atores de máxima importância nas discussões sobre câncer pelo mundo, como o francês Justin Godart (diretor da Ligue Française Contre Le Cancer), o português Francisco Gentil (diretor do Instituto Português de Oncologia), e os próprios Austin Bradford Hill e Richard Doll ${ }^{\text {xiv }}$.

Caracterizando-se como um fórum muito amplo e abordando diversos temas relacionados à doença, o congresso teve grande importância para os cancerologistas, pois mostrava às autoridades o peso que a área possuía no Brasil e o estágio de sua inserção nos fóruns internacionais. 199 médicos brasileiros, de diversos estados do país, estiveram presentes no congresso, número inferior somente à delegação dos Estados Unidos, com 300 participantes (Ibidem). A presença de autoridades como o governador de São Paulo, Lucas Nogueira Garcez, e do ministro da saúde, Mário Pinotti, também indica a relevância das discussões realizadas durante o evento.

Nas sessões temáticas e nas conferências, foi predominante o tema das novas tecnologias de tratamento da doença, principalmente o uso de variantes da mostarda nitrogenada para composição da quimioterapia, considerada à época a esperança maior, a "bala mágica" na cura do câncer (Araújo Neto \& Teixeira, 2017). Em sessões específicas, 
como de epidemiologia e câncer ginecológicos, médicos brasileiros não só apresentaram trabalhos, como coordenaram as discussões, caso de Arnaldo de Moraes, diretor do Instituto de Ginecologia do Rio de Janeiro, e Haroldo Juaçaba (diretor do Instituto do Câncer do Ceará) - na sessão de ginecologia -, e Jorge de Marsillac (médico do SNC e diretor da Fundação Napoleão Laureano - PB) - na de epidemiologia (Ibidem) .

Gradativamente, a cancerologia ganhava espaço como prática autorizada para lidar com o câncer. Alertando à população dos riscos de buscar "charlatães" e assumindo a formação de médicos para lidar com a doença na prática clínica, os cancerologistas endossavam os movimentos de ligas e associações pelo país, afirmando que o câncer era um problema de saúde importante, e somente um médico especializado poderia lidar com ele. Diferente do cenário apontado por Pickstone (2008) para os Estados Unidos, Inglaterra e França, a inovação terapêutica não parece ter sido o motor da organização da luta contra o câncer no Brasil. Naqueles países, o desenvolvimento de novas tecnologias como a radioterapia e a quimioterapia, além de sua incorporação ampla nas práticas rotineiras da medicina, conformaram arranjos profissionais e institucionais específicos.

No Brasil, o preço elevado dos equipamentos e a falta de profissionais capacitados em nível nacional para operá-los dificultavam a aquisição e uso das novas técnicas, como a quimioterapia. Na grande maioria dos casos, a aposta dos médicos seguia no modelo de cirurgias radicais, auxiliadas pela radioterapia quando disponível. Nesse sentido, a realização da detecção precoce ganhava ainda mais importância para definir a cancerologia como área específica e afirmar o papel da medicina na luta contra o câncer no Brasil. Posto isso, é plausível sugerir que o desenvolvimento da cancerologia como especialidade estava vinculado à tradução do conhecimento sobre detecção precoce na educação em saúde e na formação médica.

Esse argumento também ajuda a compreender o porquê de, nas décadas seguintes, a cancerologia não ter concentrado toda a formação médica sobre câncer, ou mesmo reunido as especialidades. À medida que as tecnologias de prevenção e detecção foram aprimoradas, e as inovações terapêuticas de fato entraram no circuito médico nacional, o movimento de especialização se abriu em vez de afunilar, criando novas especialidades (a exemplo da oncologia clínica e da mastologia) e dando mais força às já existentes (destaque para a ginecologia, a citopatologia e a urologia).

\section{A especialização das especialidades e a fragmentação prática}

Uma das pautas principais dos primeiros cancerologistas consistia na criação de 
hospitais especializados em câncer, equipados com aparelhos para diagnósticos e tratamento capazes de fornecer a "moderna assistência" aos cancerosos. Entretanto, a incorporação tecnológica era bastante cara, ocupando parte considerável dos orçamentos propostos pelas ligas e associações de combate ao câncer para seus hospitais, chegando à metade do valor previsto para uma unidade hospitalar ${ }^{\mathrm{xv}}$ (Araújo Neto, 2018a). Apesar disso, o uso de técnicas radiológicas pelos médicos era considerado algo bastante complicado, sob a alegação de que muitos profissionais não sabiam realizar a leitura dos laudos $\operatorname{radiográficos}^{\mathrm{xvi}}$.

Além do aparelho radiográfico em si, variações da tecnologia do raio-X também foram discutidas por médicos brasileiros, muitos defendendo sua inclusão à rotina clínica para aprimoramento do diagnóstico, caso da pneumomamografia, uma técnica de diagnóstico por imagem para tumores mamários ${ }^{\mathrm{xvii}}$. A incorporação de novas tecnologias para o diagnóstico de diferentes tumores foi impulsionada a partir da conformação da detecção precoce como principal estratégia para o combate ao câncer pelos especialistas e membros da campanha nacional (Araújo Neto, 2019).

O movimento de incorporação tecnológica envolvia demarcações importantes no campo médico, uma vez que cada nova ferramenta implicava práticas específicas e argumentos de autoridade sobre quem seria o profissional mais adequado para seu uso. Um caso importante desse processo foi o desenvolvimento de um modelo de detecção precoce para o câncer do colo do útero, combinando exame de visualização (colposcopia), citopatologia (teste de Papanicolaou) e cirurgia (biópsia). Esse formato específico, denominado modelo triplo, representou mais que uma reunião de tecnologias em trabalho no diagnóstico de tumores, mas também constituiu redes entre atores e instituições no campo da ginecologia brasileira, dando especificidade à especialidade no país (Teixeira \& Löwy, 2011; Lana, 2012).

Assim como a ginecologia, outras especialidades seguiram um movimento de incorporação tecnológica, o que também se relacionava a mudanças estruturais no ensino médico, cada vez mais voltado para o vetor da especialização nos anos 1950 e 1960 (Edler \& Pires-Alves, 2018). Alguns aparelhos para testes diagnósticos eram similares, possuindo especificações técnicas referentes à região do corpo para a qual eram destinados. Era o caso do gastroscópio e do colposcópio, ambos aparelhos para visualização interna do corpo que permitiam identificar úlceras em estado avançado no estômago, no caso do primeiro, e lesões pré-cancerosas na região do colo do útero, no segundo. No caso do tumor do estômago, porém, o uso dessa tecnologia era considerado somente em estágios mais 
avançados, com preferência dada ao exame bioquímico, em laboratório ${ }^{\text {xviii }}$.

Esse exemplo é ilustrativo da complexidade envolvida na abordagem do câncer pela medicina; cada tipo de tumor possui especificidades em termos de morfologia, patologia e citologia, além de demandar ações diferentes de acordo com localização, estágio de desenvolvimento e ocorrência ou não de metástase. O desenvolvimento da biomedicina ampliou as pesquisas sobre os mecanismos patológicos das neoplasias, a um só tempo trazendo mais informações aos médicos e mais incertezas (Keating \& Cambrosio, 2003). Era nesse terreno de incorporação tecnológica e ampliação do conhecimento biomédico que o projeto da cancerologia buscava se consolidar.

Em 1962, um decreto federal criou o Comitê Nacional de Ensino de Cancerologia, subordinado ao Serviço Nacional de Câncer e com o objetivo de "incentivar o ensino da cancerologia, favorecendo a participação mais efetiva e a cooperação mais eficiente dos médicos e de outros profissionais que exerçam atividades paramédicas, no combate aos tumores malignos"xix . A partir da criação do CNEC, o Instituto de Câncer passou a fornecer diplomação de cancerologista através dos cursos ministrados na instituição. A criação do Comitê era resultado das demandas dos cancerologistas na década de 1950 em torno da formalização da especialidade, e se vinculava ao modelo de ensino já implantado no Instituto desde 1953, com os cursos de cancerologia.

Em 1960, Antônio Prudente, à época possuindo grande capital simbólico na cancerologia, inclusive por ter dirigido o Serviço Nacional de Câncer nos anos 1950, indicava que o projeto unificador da especialidade não teria "necessidade e seria muito difícil", pois "não seria fácil encontrar um médico que dominasse todos os setores do conhecimento relativos aos tumores malignos" "xx. Para o cirurgião paulista, a oficialização da titulação através das escolas médicas não determinaria a existência da especialidade propriamente dita. O ponto de Prudente não era descreditar a figura do cancerologista, e sim, retomar a proposta de reunião de especialistas para a mobilização contra o câncer:

A meu ver, muito mais importante do que estabelecer, para fins de exercício
profissional, se a cancerologia é especialidade ou não, seria procurar integrar
todos os médicos na luta contra o câncer, oferecendo-lhes desde o mínimo
necessário, para que se tornem elementos de cooperação, até o máximo desejável,
para que possam dirigir a luta de uma comunidade contra os tumores malignos ${ }^{x x i}$.

A crítica de Prudente se dirigia também ao modelo de ensino médico centrado na formação especializada. Segundo ele, a formação fragmentária impedia que os futuros profisssionais tivessem uma "visão global" das interações do câncer no corpo humano, tornando o conhecimento sobre a doença demasiadamente segmentado. Além disso, 
indicava a necessidade de maiores investimentos nos institutos e hospitais, considerados uma prioridade em relação à especialidade ${ }^{\mathrm{xxii}}$.

O posicionamento do médico paulista não sintetiza o pensamento dos cancerologistas no período, mas traz elementos importantes do debate sobre especialização. Um primeiro aspecto diz respeito à insuficiência da titulação, algo ressaltado pela historiografia da especialização médica (Weisz, 2006). Apesar de compor os processos, os títulos não são definidores do sucesso das especialidades, uma vez que existem casos de especialistas sem títulos e titulados que não possuem autoridade prática, caso dos cancerologistas. Para a narrativa que traçamos aqui, essa observação é importante para que não tomemos o marco da titulação como ponto de inflexão no processo de especialização.

Outro aspecto fundamental na crítica é a vinculação entre a cancerologia e a campanha nacional contra o câncer. Se as duas eram dimensões intrínsecas na luta anticancerosa em seu início, um gradual afastamento pode ser percebido a partir dos anos 1960. Embora não tenhamos um argumento para tentar compreender esse fenômeno, é possível supor, a partir de falas como a de Prudente citada acima, que houve uma diferenciação de objetivos. O projeto de especialidade da cancerologia foi se concentrando cada vez mais na demarcação de autoridade e prática profissional, enquanto a mobilização pela campanha nacional tinha como mote central as demandas por uma efetiva nacionalização, ou seja, a criação de institutos e hospitais em todos os estados do país (Araújo Neto, 2019).

Enquanto isso, antigas e novas especialidades ganhavam território no cuidado aos cancerosos, caso da mastologia. A organização do campo profissional ligado ao câncer de mama se inicia com a criação da Sociedade Brasileira de Patologia Mamária, em 1959, almejando a transformação da mastologia em especialidade médica. A partir da década de 1970, então renomeada de Sociedade Brasileira de Mastologia (SBM), passou a contar com regionais em diversos estados do país, oferecendo formação continuada a cirurgiões e ginecologistas, e com o objetivo de estimular ações públicas e privadas para o controle do câncer de mama no país (Teixeira \& Araújo Neto, 2020b).

Com a ampliação e especialização das especialidades, associadas também ao processo de conformação do mercado de saúde no Brasil, o projeto de uma especialidade que reunisse todos os conhecimentos e práticas na luta contra o câncer gradativamente perdeu espaço na agenda médica. Mesmo a oncologia, que ganhou força no país de maneira mais efetiva a partir dos anos 1980, não possuía esse objetivo de unificação dos especialistas, demarcando sua prática a partir da compreensão clínica da doença, mas 
articulando outras especialidades nas ações de cuidado.

Ainda no fim dos anos 1960 e início dos anos 1970, são encontrados registros de formação profissional em cancerologia, caso dos Congressos Integrados de Cancerologia, organizados pela Sociedade Brasileira de Cancerologia (SBC), em parceria com a Sociedade Latino-Americana de Quimioterapia Antineoplásica, a Sociedade Brasileira de Radioterapia, e Rede Nacional Feminina Contra o Câncer ${ }^{\text {xxiii }}$. Entretanto, esses congressos integrados possuíam caráter de aperfeiçoamento e capacitação profissional, não de especialização. Embora a pauta da titulação ainda ocupasse uma parcela das mobilizações $^{\text {xxiv }}$, a figura do cancerologista ocupou cada vez menos espaço na arena médica e da saúde pública nos anos 1970.

Isso não significou, porém, o desuso do termo cancerologia, que passou a ser utilizado principalmente em dois sentidos. $\mathrm{O}$ primeiro se referia ao ensino nas faculdades de medicina, a partir da demanda por disciplinas específicas para o câncer na graduação e pós-graduação ${ }^{\mathrm{xxv}}$, enquanto o segundo consistia numa nomenclatura ampla para o campo da medicina do câncer. De especialista com maior capacidade para lidar com os tumores, a figura do cancerologista passou a descrever os personagens atuantes nos institutos de câncer e na campanha nacional. Nas reminiscências semânticas, a sociedade e o periódico mantiveram o nome "cancerologia" (Sociedade Brasileira e Revista Brasileira de Cancerologia), associada aos próprios profissionais da área como um elemento da história da luta contra o câncer no país.

\section{Considerações finais}

A história da cancerologia consiste em um caso interessante para a historiografia, tanto pelo sentido do seu projeto quanto pelo eventual desfecho que teve. Em termos de como foi projetada a especialidade, é interessante observar que os cancerologistas almejavam uma "especialização generalista", o que poderia soar como uma contradição em termos, mas possuía uma lógica plausível à configuração do conhecimento médico sobre o câncer em meados do século XX e à organização institucional do combate à doença no país. Quanto ao enfraquecimento do projeto de especialidade, é curioso observar também como o desenvolvimento da biomedicina, com novas tecnologias, práticas e saberes, foi um fator fundamental para que a cancerologia não vingasse, descontruindo a visão tradicional de que especialização é resultado de avanço da medicina.

Outro aspecto explorado neste artigo e que possui implicações mais amplas para a história da especialização diz respeito à vinculação entre papéis sociais e especialidades 
profissionais. Na primeira dimensão, os cancerologistas estabeleceram para si uma figura com moldes específicos e um objetivo claro: organizar o combate ao câncer no Brasil; nesse aspecto, a cancerologia consolidou modelos institucionais de atenção oncológica (Araújo Neto, 2019) e trajetórias profissionais. Os cancerologistas da primeira geração ocupam um lugar destacado nas produções de memória sobre o controle do câncer no país. Na segunda dimensão, porém, a cancerologia não possuiu a mesma credibilidade, tanto pelas limitações do projeto unificador quanto pela força das outras especialidades.

O eventual abandono da cancerologia como especialidade não significou o fim de sua conotação como movimento para controle do câncer, e mesmo a sua sobrevivência institucional através da Sociedade e de seus congressos, além da Revista Brasileira de Cancerologia. Ainda cabe discutir, nos meandros do processo de especialização médica, como o desenvolvimento do mercado de saúde no Brasil, principalmente na segunda metade do século XX, colocou pautas específicas oriundas do setor privado. A ampliação da medicina hospitalar e o fortalecimento dos grupos empresariais estabeleceram arranjos institucionais e práticas de cuidado, a exemplo da incorporação da mamografia (Teixeira \& Araújo Neto, 2020a). Por esse viés, será possível discutir a emergência da oncologia e o fortalecimento de especialidades como a mastologia.

\section{Referências}

ARAÚJO NETO, Luiz Alves e TEIXEIRA, Luiz Antonio. De doença da civilização a problema de saúde pública: câncer, sociedade e medicina brasileira no século XX. Boletim do Museu Paraense Emílio Goeldi. Ciências Humanas, v. 12, n.1, p. 173-188, jan-abr, 2017.

ARAÚJO NETO, Luiz Alves. "Uma doença brasileira? Antônio Prudente e a luta contra o câncer no Brasil". In: CARVALHO, Leonardo \& BARCHI, Felipe. Intelectuais e Nação: leituras de Brasil na República. Curitiba: Editora Appris, 2018b. p. 227-242.

ARAÚJO NETO, Luiz Alves. O problema do câncer no Ceará: cancerologia, controle do câncer e a atividade coletiva da medicina. Rio de Janeiro: Luminária Acadêmica, 2018a.

ARAÚJO NETO, Luiz Alves. Prevenção do câncer no Brasil: mudança conceitual e continuidade institucional no século XX. Tese de Doutorado. Programa de Pós-Graduação em História das Ciências e da Saúde - Casa de Oswaldo Cruz/Fiocruz. Rio de Janeiro, 2019.

DACHEZ, Roger. Histoire de la Médecine: de l'Antiquité au $\mathrm{XX}^{\mathrm{e}}$ siècle. Paris: Tallandier, 2004.

EDLER, Flávio \& PIRES-ALVES, Fernando. “A educação médica: do aprendiz ao especialista”. In: TEIXEIRA, Luiz Antonio; PIMENTA, Tânia Salgado; HOCHMAN, 
Gilberto. História da Saúde no Brasil. São Paulo: Editora Hucitec, 2018. p. 101-144.

HAYTER, Charles. An Element of Hope: Radium and the Response to Cancer in Canada, 1900-1940. Toronto: McGill-Queen's University Press, 2005.

JAIN, Lochlann. Malignant: How cancer becomes us. Berkeley: University of California Press, 2013.

JAISON, Marie. O estudo de práticas médicas: o cenário da sociologia das profissões. Saúde e Sociedade, São Paulo, v. 27, n. 3, p. 704-714, 2018.

KEATING, Peter e CAMBROSIO, Alberto. Biomedical Platforms. Realigning the normal and the pathological in late-twentieth century medicine. Cambridge/London: The MIT Press, 2003.

LANA, Vanessa. Ferramentas, práticas e saberes: a formação de uma rede institucional para a prevenção do câncer do colo do útero no Brasil (1936 - 1970). Tese de doutoramento: Programa de Pós-Graduação em História das Ciências e da Saúde. Casa de Oswaldo Cruz, Rio de Janeiro, 2012.

PASVEER, Bernike. Knowledge of shadows: the introduction of X-ray images in medicine. Sociology of Health \& Illness. Vol. 11, nº 4, 1989. p. 360-381.

PICKSTONE, John V. "Contested Cumulations: Configurations of Cancer Treatment through the Twentieth Century". In: CANTOR, David (Ed.). Cancer in the Twentieth Century. Baltimore: John Hopkins University Press, 2008.

PINELL, Patrice. Análise Sociológica das Políticas de Saúde. Rio de Janeiro: Editora Fiocruz, 2010.

ROSENBERG, Charles E. The Tiranny of Diagnosis: Specific Entitites and Individual Experience. The Milbank Quarterly, v. 80, n. 2, 2002. p. 237-260.

TEIXEIRA, Luiz Antonio e ARAÚJO NETO, Luiz Alves. Câncer de mama no Brasil: medicina e saúde pública no século XX. Saúde e Sociedade, v. 29, n.3, p. 1-12, 2020 b. TEIXEIRA, Luiz Antonio e ARAÚJO NETO, Luiz Alves. Still controversial: Early detection and screening for breast cancer in Brazil, 1950-2010s. Medical History, v. 64, n.1, 2020a, p. 52-70.

TEIXEIRA, Luiz Antonio e FONSECA, Cristina. De doença desconhecida a problema de saúde pública: o INCA e o controle do câncer no Brasil. Rio de Janeiro: Ministério da Saúde, 2007.

TEIXEIRA, Luiz Antonio e LÖWY, Ilana. Imperfect tools for a dificult job: colposcopy, 'colpocitology' and screening for cervical cancer in Brazil. Social Studies of Science, 41: 585 - 608, July, 2011.

TEIXEIRA, Luiz Antonio. O câncer na mira da medicina brasileira. Revista Brasileira de História da Ciência. Rio de Janeiro, v. 2, n. 1, p. 104-117, jan - jun 2009.

TEIXEIRA, Luiz Antonio. O controle do câncer no Brasil na primeira metade do século 
XX. História, Ciências, Saúde - Manguinhos. Rio de Janeiro: Vol. 17, Supl. 1, 2010.

WEISZ, George. Divide and conquer: a comparative history of medical specialization. Oxford: Oxford University Press, 2006.

WEISZ, George. The Emergence of Medical Specialization in the Nineteenth Century. Bulletin of History of Medicine. 2003, 77: 536

Recebido em 29/07/2020

Aprovado em 01/09/2020

Publicado: $1 \% 01 / 2021$

Fomento/Financiamento: Este artigo é resultado de pesquisa financiada pela Fundação Carlos Chagas de Amparo à Pesquisa do Estado do Rio de Janeiro (FAPERJ), pelos seguintes fomentos: Cientista do Nosso Estado (Luiz Antonio Teixeira); Pós-Doutorado Nota 10 (Luiz Alves Araújo Neto); e Projeto Humanidades.

\footnotetext{
i Graduado em História, com mestrado e doutorado em História das Ciências e da Saúde, realizados no Programa de Pós-Graduação em História das Ciências e da Saúde da Casa de Oswaldo

Cruz/Fiocruz.Professor colaborador do Programa de Pós-graduação em História das Ciências e da Saúde da FIOCRUZ.

ii Historiador, mestre em Saúde Coletiva e doutor em História Social. É professor permanente do Programa de Pós-Graduação em Saúde da Criança e da Mulher - PPGSCM do Instituto Fernandes Figueira - Fiocruz, do Programa de Saúde da Família da Universidade Estácio de Sá e do Programa de Pós-Graduação em Divulgação da Ciência, Tecnologia e Saúde da Casa de Oswaldo Cruz. Também atua como professor colaborador no Programa de Pós-graduação em História das Ciências e da Saúde da Casa de Oswaldo Cruz Fiocruz. Sua pesquisa mais recente concentra-se na medicalização do parto.

iii A ideia de doença local se refere à origem das enfermidades em tecidos ou órgãos do corpo, seja por uma irritação constante/lesão ou pela atuação de um agente patogênico. Essa perspectiva, introduzida pela anatomoclínica no século XIX e fortalecida pela microbiologia, se contrapôs a percepções constitucionalistas das doenças, que consideravam o adoecimento como a manifestação local de um problema geral do corpo. Ver Rosenberg, 2002.
}

iv KROEFF, Mário. O ensino da cancerologia deve ser ministrado nas universidades? Revista Brasileira de Cancerologia. Vol. 6, no 9. Dezembro, 1952; GRAÇA, Döllinger da. O problema do câncer no Brasil: observação pessoal no câncer da mama e do útero. Brazil Medico, 32/33: 298—304. 1944.

\footnotetext{
${ }^{\vee}$ Vale destacar que o discurso dos cancerologistas sobre os perigos dos charlatães se pautava muito mais numa retórica do que propriamente em uma maior eficiência no cuidado aos doentes de câncer. Essa projeção de certezas dos médicos sobre os tumores é chamada por Lochalann Jain (2001) de "aparência de conhecimento", um recurso para criação de autoridade.
}

vi PRUDENTE, Antônio. O câncer precisa ser combatido. São Paulo: Calvino Filho Editor, 1934; KROEFF, Mário. O câncer como flagelo. Revista Brasileira de Cancerologia. Vol. 4, nº 7. Jan - Jun, 1951.

vii RABELLO, Eduardo. O problema do câncer. In: CONGRESSO BRASILEIRO DE HYGIENE, 2., 1924, Belo Horizonte. Rio de Janeiro: Pimenta de Mello e Cia., 1928. v. 1.

viii KROEFF, Mário. "O papel da electro-cirurgia numa campanha anti-cancerosa". Anais do Primeiro Congresso Brasileiro de Câncer. Actas e Trabalhos. 2 volumes. Promovidos pela Sociedade de Medicina e Cirurgia do Rio de Janeiro. 1936. 
${ }^{i x}$ PRUDENTE, Antonio. Critério atual no tratamento do câncer de mama. Revista Brasileira de Cancerologia. Ano 1, vol. 1, 1947. p. 6-29.

${ }^{x}$ KROEFF, Mário. “O papel da electro-cirurgia numa campanha anti-cancerosa”. Anais do Primeiro Congresso Brasileiro de Câncer. Actas e Trabalhos. 2 volumes. Promovidos pela Sociedade de Medicina e Cirurgia do Rio de Janeiro. 1936; PRUDENTE, Antônio. O câncer precisa ser combatido. São Paulo: Calvino Filho Editor, 1934.

xi KROEFF, Mário. Apresentação. Revista Brasileira de Cancerologia. Volume 1, Número 1. Setembro, 1947. p. 1.

xii KROEFF, Mário. O ensino da cancerologia deve ser ministrado nas universidades? Revista Brasileira de Cancerologia. Vol. 6, nº 9. Dezembro, 1952, p. 89.

xiii RBC (Revista Brasileira de Cancerologia). Cursos S.N.C. Revista Brasileira de Cancerologia. Vol. 12, $\mathrm{n}^{\circ}$ 13. Junho, 1955, p. 104.

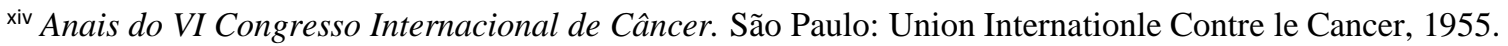

xv Documentos sobre câncer (...). Arquivo Gustavo Capanema. GC h1938.02.05. Série: h- Ministério da Educação e Saúde - Saúde e serviço social. Rolo 66 fot. 821 a 1027.

${ }^{x v i}$ BURNIER, Egberto Moreira Penido. Dos tumores ósseos. Revista Brasileira de Cirurgia. Ano X, $\mathrm{n}^{\circ} 10$. Outubro, 1941. p. 53.

xvii COUTINHO, Alberto. Cancer da Mama. Annaes de Ginecologia. Ano VI, 1941. Fevereiro, vol. 11. p. 218-219.

xviii GALIZZI, João. Diagnóstico do câncer do estomago. Brasil Medico. Ano LVIII, n. 4-5. Janeiro, 1944. p. 19-21.

${ }^{\text {xix }}$ Brasil. Decreto $n^{\circ} 1.100$, de 30 de maio de 1962. Cria o "Comitê Nacional de Ensino de Cancerologia", como órgão assessor do Serviço Nacional de Câncer e aprova seu Regimento. Disponível em: www.planalto.gov.br/legislacao. Acesso em: 17/08/2017.

${ }^{x x}$ PRUDENTE, Antônio. Deve a cancerologia ser considerada uma especialidade médica? Boletim de Oncologia, v. 3, n.1, 1960, p. 67-68.

xxi Ibidem, p. 67.

xxii Ibidem, p. 68 .

xxiii BARRETO LINS, José Roberto. Congressos Integrados de Cancerologia. Boletim de Oncologia, v. 57, n. $1-2,1969$, p. 41.

xxiv AGOSTINI, Alexandre. A Cancerologia como especialidade. Boletim de Oncologia, v, 52, n.6, 1966, p. 365 .

${ }^{x x v}$ ARAÚJO CINTRA, Renato. Ensino da Cancerologia. Boletim de Oncologia, v. 54, n. 5-6, 1967, p. 177. 